\title{
Individual differences in sequence learning and auditory pattern sensitivity as revealed with evoked potentials
}

\author{
Elisabet Tubau, ${ }^{1,2}$ Carles Escera ${ }^{2}$ Vanessa Carral ${ }^{2}$ and María-José Corral ${ }^{2}$ \\ ${ }^{1}$ Department of Basic Psychology, Faculty of Psychology, University of Barcelona, Pg de la Vall d'Hebron, 171, 08035 Barcelona, \\ Catalonia, Spain \\ ${ }^{2}$ Cognitive Neuroscience Research Group, Department of Psychiatry and Clinical Psychobiology, Faculty of Psychology, \\ University of Barcelona, 08035 Barcelona, Catalonia, Spain
}

Keywords: action control, auditory action effects, explicit and non-explicit learners, mismatch negativity, sequential action

\begin{abstract}
Research on motor sequence acquisition has shown significant differences between learners. Learners who develop explicit knowledge respond faster than non-explicit ones and they show larger amplitude in event-related brain potentials to sequence deviants. There is evidence that memory span correlates with the amount of sequence learned, but the specific mechanisms subserving such differences are still unknown. Recently, it has been observed that performance of explicit learners, but not of nonexplicit ones, improves when presented with auditory action effects. Accordingly, differences between learners might be related to differences in auditory rhythm perception. To test this hypothesis, the mismatch negativity (MMN)-evoked potential elicited to stimuli violating stimulus alternation (i.e. low pitch, high pitch) was recorded in explicit and non-explicit sequence learners. Results confirmed our prediction: explicit learners showed larger amplitude of the MMN to the violation of the auditory rhythm, suggesting new theoretical implications to account for individual differences in sequential action control.
\end{abstract}

\section{Introduction}

Sequential activities, such as music or sports, require controlling both the order of the actions and the precise timing in which they have to be performed. The problem of how serial order is acquired and represented in the brain (Lashley, 1951) has nowadays received special interest in the serial reaction time (RT) task. In this task, a sequence of button presses has to be carried out as responses to certain mandatory stimuli (e.g. visual signals at corresponding spatial locations). By repeating the sequence, responses are performed faster, especially if participants learn the sequential pattern explicitly (e.g. Hoffmann \& Koch, 1997; Eliassen et al., 2001; Rüsseler et al., 2003; Tubau \& López-Moliner, 2004).

Interestingly, several studies have shown that sequential performance improves if each response determines the occurrence of a specific sound (Hoffmann et al., 2001; Stöcker \& Hoffmann, 2004; Tubau et al., 2007), suggesting that response-contingent sounds enhance the chunking of individual actions into larger units as in rhythmic melodies. However, Tubau et al. (2007) showed that auditory action effects did not facilitate the acquisition of the serial order but the precise timing control. That is, the percentage of participants who learned the order (explicit learners) was the same with and without auditory effects, but performance of explicit learners, differing from non-explicit ones, improved when presented with response-contingent sounds (Tubau et al., 2007). Could these individual differences be related to differences in neural representation of auditory rhythms?

Correspondence: Dr E. Tubau, ${ }^{1}$ Department of Basic Psychology, as above. E-mail: etubau@ub.edu

Received 26 February 2007, revised 14 May 2007, accepted 17 May 2007
The present study aimed at answering this question using eventrelated brain potentials (ERP). For that purpose, we used the MMN auditory ERP (Näätänen \& Escera, 2000), which has been proposed as a good indicator of the accuracy of central sound representation (Näätänen \& Winkler, 1999) and, accordingly, of behavioural perceptual performance (i.e. Tiitinen et al., 1994; Amenedo \& Escera, 2000). The MMN is elicited, with no task requirements, by occasionally 'deviant' sounds occurring in a sequence of repetitive ('standard') stimuli (Näätänen et al., 2001). When the brain's response to the standard stimuli is subtracted from that to the deviant one, the MMN can be seen at 100-200 ms from stimulus-change onset. The MMN can be elicited by any perceptible physical change, i.e. pitch or duration, or by contrasting more complex sound features such as abstract invariances (Carral et al., 2005), tone patterns (Atienza et al., 2003) and phonemes (Näätänen et al., 1997). Critically, the brain's neurophysiological response to deviant stimuli implies that a neural representation of the preceding repetitive stimulus features has been formed (Näätänen \& Winkler, 1999; Näätänen et al., 2001). Hence, if motor sequence acquisition and auditory rhythm perception correlate, MMN differences between explicit and non-explicit learners should be observed. Specifically, we expected that learners showing better timing control of the motor sequence (explicit group) would show larger MMN responses to auditory pattern deviants than non-explicit learners.

\section{Materials and methods}

We replicated one of the sequence learning conditions of Tubau et al. (2007), which showed high differences between explicit and nonexplicit learners regarding their sensitivity to auditory action effects. 
Eighteen students at the University of Barcelona were presented with an incidental learning task. Participants were instructed to respond as fast and as accurately as possible, pressing a left or right button according to the spatial location of a visual mark (X) on the screen. The stimulus location followed six continuous repetitions per block of the sequence: right-left-right-right-left-left-right-left, unknown by the participants. Responses were executed with the left and right index for left and right buttons, respectively. The stimulus was on the screen until response. The temporal interval between the response and the next stimulus was $250 \mathrm{~ms}$. Additionally, different $100-\mathrm{ms}$ tones $(200 \mathrm{~Hz}$ and $440 \mathrm{~Hz})$ were presented as effects of left and right responses, respectively. Participants were informed that the sounds were irrelevant and that they had to respond only to the location of the X. After the learning task (formed by 11 blocks), participants were required to reproduce, without the stimuli, the sequential pattern both by means of the same buttons and verbally.

In a different session, ERPs of these participants were recorded in order to measure the brain sensitivity to auditory pattern violations. According to the Declaration of Helsinki, informed consent was obtained from each subject, after the nature of the study was explained to them. Participants were presented with a paradigm of pitch alternation similar to that developed by Ford \& Hillyard (1981) and modified by Atienza et al. (2003; experiment 1). The stimulation consisted of two blocks of 100-stimulus trains (4800 tones altogether, delivered by Stim stimulation system from Neuroscan, Herndon, VA, USA). Each train was formed by 24 alternating pure tones of either 500 or $1000 \mathrm{~Hz}$. Tones lasted $30 \mathrm{~ms}$, including $5 \mathrm{~ms}$ of rise/fall times, and were presented binaurally through headphones at an intensity of $80 \mathrm{~dB}$ (SPL). Tones within the train were delivered at a constant stimulus-onset asynchrony of $130 \mathrm{~ms}$, which was also the inter-train interval. Stimulus trains were presented in a predictable way (LHLHLHHLHLHLHLHLHLLHLHLH), in which the ' $L$ ' and ' $H$ ' represent the two tone frequencies used in the experiment. The ' $\mathbf{L}$ ' and ' $\mathbf{H}$ ' denote the deviant events (the repetition of a tone within the train), whereas the ' $L$ ' and ' $H$ ' correspond to the standard tones used for comparison. There were altogether 400 deviant events. The electroencephalogram was continuously recorded $(\mathrm{A} / \mathrm{D}$ ratio $=500 \mathrm{~Hz}$; band-pass filter $=0.1-30 \mathrm{~Hz}$ ) with SynAmps amplifiers (NeuroScan) from electrodes placed at the following locations: Fp1, Fp2, F3, F7, Fz, F4, F8, C3, Cz, C4, T3, T4, T5, T6, P3, Pz, P4, and the left (LM) and right (RM) mastoids. An electrode attached to the outer canthus of the left eye served to monitor the EOG, while participants watched a silent video-movie. The common reference electrode was placed on the tip of the nose. Epochs with EOG activity (whenever the standard deviation within a $200-\mathrm{ms}$ interval exceeded $\pm 30 \mu \mathrm{V}$ at any recording site) as well as the first five trials of each block were rejected. For each trial, an epoch of $930 \mathrm{~ms}$, including a prestimulus baseline of $150 \mathrm{~ms}$, was selected for averaging. Detrend correction was applied to each individual grand average waves. The MMN was evaluated as the mean amplitude in a 60-ms latency window (310-370 ms) of the difference wave resulting from subtracting the standard trial ERPs from those elicited to the deviant trials.

\section{Results}

Participants were assigned to the Explicit group when they reproduced the complete sequence (elements and order) correctly (correlation between verbal and motor reproduction of the sequence was 1). As the sequence was presented continuously within a block, any starting point was considered correct (i.e. RRLLRLRL; RLRLRRLL; RLRRLLRL). Otherwise, they were included in the Non-explicit group. The percentage of explicit learners was $50 \%$ (nine out of 18).

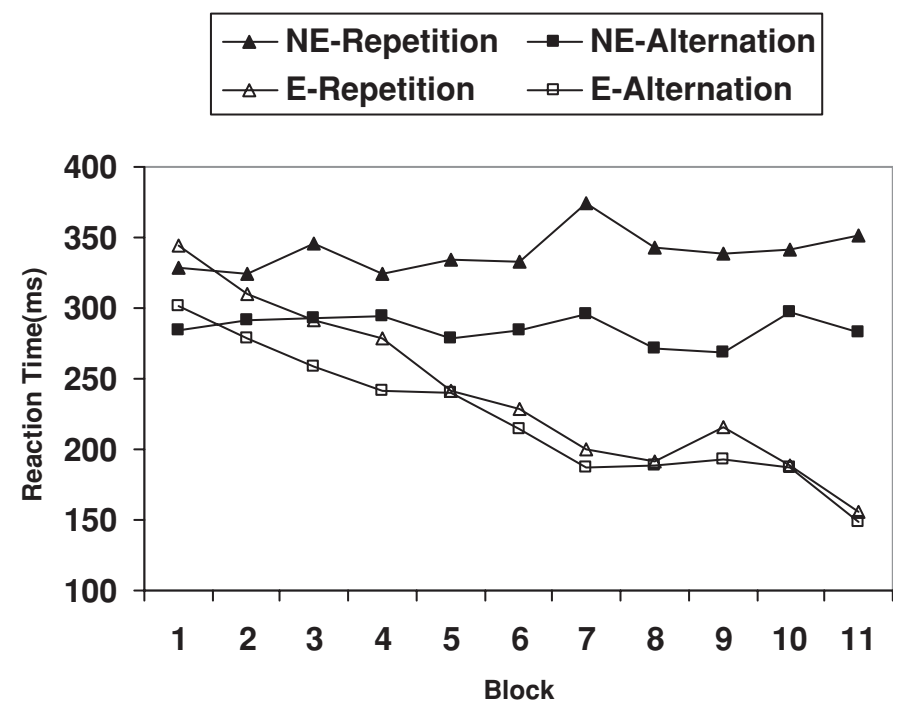

FIG. 1. Response time means (ms) through blocks of trials split by group and type of transition in the sequence learning task (E, explicit group; NE, nonexplicit group).

Results of the sequence learning task replicated the ones previously observed (Tubau et al., 2007; experiments 5A and 5B). Figure 1 shows RT data for correct responses (the percentage of errors was $2.5 \%$ ), split by group and type of transition (alternation and repetition). Notice that in the repeating sequence alternations are three times more frequent than repetitions, and this frequency bias differentiated non-explicit from explicit participants (Tubau \& LópezMoliner, 2004; Tubau et al., 2007). An ANOVA with Block (11 sequence blocks) and Transition (alternation and repetition) as withinparticipants factors, and Group (explicit and non-explicit) as a between-participant one showed that all the main factors were significant: Block $\left(F_{10,160}=10.01, P<0.001, \eta^{2}=0.39\right)$; Transition $\left(F_{1,16}=23.47, \quad P<0.001 ; \quad \eta^{2}=0.59\right) ; \quad$ and Group $\left(F_{1,16}=13.18, P<0.01 ; \eta^{2}=0.45\right)$. Group $\times$ Block and Group $\times-$ Block-Transition interactions were also significant $\left(F_{10,160}=12.42\right.$, $P<0.001, \eta^{2}=0.44 ; F_{10,160}=2.94, P<0.01, \eta^{2}=0.15$, respectively). Analyses for each group separately showed that whereas neither Block nor Block $\times$ Transition were significant for non-explicit participants $(F<1)$, the explicit group showed a significant Block $\left(F_{10,80}=16.78, P<0.001, \eta^{2}=0.68\right)$ and Block-Transition interaction $\left(F_{10,80}=2.85, P<0.01, \eta^{2}=0.26\right)$. As can be seen in Fig. 1, explicit participants responded much faster and equally fast for repetitions and alternations in the last blocks. In contrast, RT of nonexplicit participants did not vary through blocks of trials and was always affected by the transitional frequency (alternations were always performed faster than repetitions; $F_{1,8}=13.48, P<0.01, \eta^{2}=0.63$ ).

ERP data from two participants of the explicit group were discarded due to excessive artefacts during the recordings. Therefore, the final ERP sample included seven participants from the explicit group and nine (all of them) from the non-explicit group. The ANOVA with MMN amplitudes, with group as between-participant factor (Explicit, Nonexplicit) and electrode as within-participant factor (Fp1, F3, Fz, F4, $\mathrm{C} 3, \mathrm{Cz}, \mathrm{C} 4, \mathrm{P} 3, \mathrm{Pz}, \mathrm{P} 4)$, showed that the MMN was clearly elicited in the two experimental groups but it was of significant smaller amplitude in the non-explicit group $\left(F_{1,14}=4.634 ; P<0.05\right)$. The electrode variable was significant $\left(F_{1,9}=4.683, P<0.05\right)$, indicating that the MMN was larger over the anterior electrodes but it did not interact with the group factor (Fig. 2). 


\section{Pattern MMN}
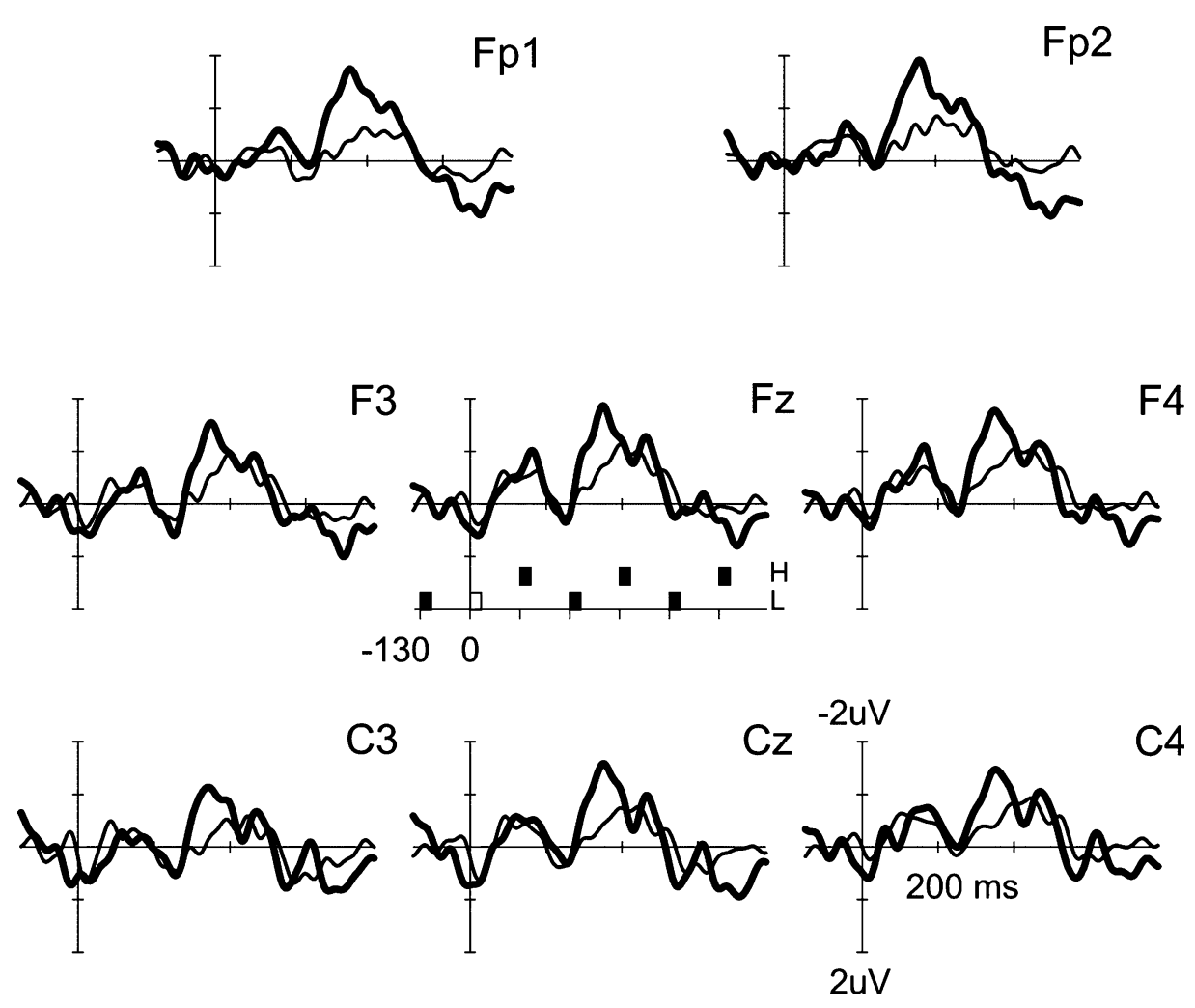

\section{- Explicit group \\ - Non-explicit group}

FIG. 2. The mismatch negativity (MMN) elicited to auditory pattern violation in explicit (thick) and non-explicit (thin) sequence learners. Notice that an example of the stimulus sequence is represented under the Fz waveform: L, low-frequency tone; H, high-frequency tone; the white square at time zero represents the deviant stimulus, i.e. stimulus repetition breaking tone alternation.

\section{Discussion}

The present study is a first attempt to apply ERP data to the analyses of individual differences in motor sequence learning. Specifically, we found a new relationship between sensitivity to auditory rhythms (measured as MMN response to auditory pattern violations) and amount of learning in a motor sequential task. Participants who showed a high degree of sequence learning (measure both as changes in RT through the learning blocks and as amount of explicit knowledge) also showed a large MMN response to auditory pattern deviants. In contrast, the MMN response of poor sequence learners was much less pronounced. As introduced, only the performance of good learners (the explicit group) was positively affected by the auditory action effects presented incidentally in the learning task (Tubau et al., 2007), suggesting that good sequence learners are more sensitive to certain auditory information. Results of the analyses of ERPs confirmed this hypothesis; good sequence learners seem to form auditory patterns more accurately than poor learners.

Previous studies using ERP data (e.g. Eimer et al., 1996; Baldwin \& Kutas, 1997; Rüsseler et al., 2003) have also shown differences between explicit and non-explicit learners, but during the sequence learning process. That is, ERP recordings have been used as further evidence about the correspondence between electrophysiological, behavioural and self-reported measures. In these studies, ERPs to standard elements of a repeating sequence (i.e. a series of letters mapped to specific motor responses) are compared with ERPs to deviant elements. A systematic larger amplitude for the N2b- and P3bcomponents for deviant elements has been observed only in the case of explicit learners (Baldwin \& Kutas, 1997; Rüsseler et al., 2003). Only explicit learners also showed enhanced lateralized readiness potential for incorrect but predicted responses (Eimer et al., 1996), suggesting that these participants were anticipating and preparing in advance the response. Such differences between explicit and non-explicit learners validate the use of self-reported knowledge as criteria for grouping participants. However, these studies cannot reveal specific mechanisms that might explain why some participants are better or faster in sequential learning tasks.

On the other hand, studies on individual differences have found significant correlations between verbal memory span and amount of sequence learning (i.e. Frensch \& Miner, 1994; Unsworth \& Engle, 2005). However, such correlations have been observed only in intentional learning conditions and, as shown in the present experiment, large individual differences can also be observed when the participants' goal is just to react to the stimuli. Furthermore, it is still 
unknown which specific memory mechanisms contribute to such correlations (but see Saito, 2001 for interesting suggestions about the relationship between memory of auditory rhythms and timing control mechanisms in the phonological loop).

Certainly, the intention to learn would have promoted the acquisition of the simple sequence used in the experiment (about $90 \%$ of the participants were in the explicit group when presented with intentional instructions; Tubau et al., 2007), reflecting the active involvement of the phonological loop or subvocal rehearsal mechanisms. Nevertheless, the fact that some participants were positively affected by irrelevant auditory effects in an incidental learning task suggests that more automatic memory mechanisms should also have a role. The observed relation between sensitivity to auditory patterns and motor sequence performance does not allow establishing a causal connection between both abilities, but the facility of auditory material for representing rhythmic patterns (Burgess \& Hitch, 1999) suggests such a possibility.

As introduced, a critical component of sequential action is the precise control of timing. Indeed, motor skilled performance develops by consolidating specific rhythmic movements (Sakai et al., 2004). Learning, thus, is enhanced if the rhythmic structure (i.e. relevant chunks, temporal intervals) can be easily perceived, i.e. by means of auditory signals. Although more research is needed for establishing clear causal connections, our results are coherent with the specific role of auditory rhythm perception in timing control processes. If such causal connection was confirmed, it would imply that not only executive attention is responsible for individual differences in higher order cognition (Unsworth \& Engle, 2005); certain preattentive processes (i.e. auditory pattern representation) may also determine performance in complex motor skills.

\section{Acknowledgements}

We thank Áurea Moreno for her help in data collection, and Juan Bakri for his help in data analysis. This study was supported by the Spanish Ministry of Education and Science (SEJ2006-00496/PSIC) and the Generalitat de Catalunya (SGR2005-00953).

\section{Abbreviations}

ERP, event-related potential; MMN, mismatch negativity; RT, reaction time.

\section{References}

Amenedo, E. \& Escera, C. (2000) The accuracy of sound duration representation in the human brain determines the accuracy of behavioural perception. Eur. J. Neurosci., 12, 2570-2574.

Atienza, M., Cantero, J.L., Grau, C., Gómez, C., Domínguez-Marín, E. \& Escera, C. (2003) Effects of temporal encoding on auditory object formation: a mismatch negativity study. Cogn. Brain Res., 16, 359-371.
Baldwin, K.B. \& Kutas, M. (1997) An ERP analysis of implicit structured sequence learning. Psychophysiology, 34, 74-86.

Burgess, N. \& Hitch, G.J. (1999) Memory for serial order: a network model of the phonological loop and its timing. Psychol. Rev., 106, 551-581.

Carral, V., Corral, M.J. \& Escera, C. (2005) Auditory event-related potentials as a function of abstract change magnitude. Neuroreport, 16, 301-305.

Eimer, M., Goschke, T., Schlaghecken, F. \& Stürmer, B. (1996) Explicit and implicit learning of event sequences: evidence from event-related brain potentials. J. Exp. Psychol. Learn. Mem. Cogn., 22, 970-987.

Eliassen, J.C., Souza, T. \& Sanes, J.N. (2001) Human brain activation accompanying explicitly directed movement sequence learning. Exp. Brain Res., 141, 269-280.

Ford, J.M. \& Hillyard, S.A. (1981) Event-related potentials (ERPs) to interruptions of a steady rhythm. Psychophysiology, 18, 322-330.

Frensch, P.A. \& Miner, C.S. (1994) Individual differences in short-term memory capacity on an indirect measure of serial learning. Mem. Cogn., 22, 95-110.

Hoffmann, J. \& Koch, I. (1997) Stimulus-response compatibility and sequential learning in the serial reaction time task. Psychol. Res., 60, 87-97.

Hoffmann, J., Sebald, A. \& Stoecker, C. (2001) Irrelevant response effects improve serial learning in SRT tasks. J. Exp. Psychol. Learn. Mem. Cogn., 27, 470-482

Lashley, K.S. (1951) The problem of serial order in behavior. In Jeffress, L.A. (Ed.), Cerebral Mechanisms in Behavior. Wiley, New York, pp. 112136.

Näätänen, R. \& Escera, C. (2000) Mismatch negativity: clinical and other applications. Audiol. Neurootol., 5, 105-110.

Näätänen, R., Lehtokoski, A., Lennes, M., Cheour, M., Huotilainen, M., Iivonen, A., Vainio, M., Alku, P., Ilmoniemi, R.J., Luuk, A., Allik, J., Sinkkonen, J. \& Alho, K. (1997) Language-specific phoneme representations revealed by electric and magnetic brain responses. Nature, $\mathbf{3 8 5}, 432$ 434.

Näätänen, R., Tervaniemi, M., Sussman, E., Paavilainen, P. \& Winkler, I. (2001) 'Primitive Intelligence' in the auditory cortex. Trends Neurosci., 24, 283-288.

Näätänen, R. \& Winkler, I. (1999) The concept of auditory stimulus representation in cognitive neuroscience. Psychol. Bull., 125, 826-859.

Rüsseler, J., Henninghaussen, E., Münte, T. \& Rösler, F. (2003) Differences in incidental and intentional learning of sensorimotor sequences as revealed by event-related brain potentials. Cogn. Brain Res., 15, 116-126.

Saito, S. (2001) The phonological loop and memory for rhythms: an individual differences approach. Memory, 9, 313-322.

Sakai, K., Hikosaka, O. \& Nakamura, K. (2004) Emergence of rhythm during motor learning. Trends Cogn. Sci., 8, 547-553.

Stöcker, C. \& Hoffmann, J. (2004) The ideomotor principle and motor sequence acquisition: tone effects facilitate movement chunking. Psychol. Res., 68, 126-137.

Tiitinen, H., May, P., Reinikainen, K. \& Näätänen, R. (1994) Attentive novelty detection in humans is governed by pre-attentive sensory memory. Nature, 372, 90-92.

Tubau, E., Hommel, B. \& López-Moliner, J. (2007) Modes of executive control in sequence learning: from stimulus-based to plan-based control. J. Exp. Psychol. Gen., 136, 43-63.

Tubau, E. \& López-Moliner, J. (2004) Spatial interference and response control in sequence learning: the role of explicit knowledge. Psychol. Res., 68, $55-63$.

Unsworth, N. \& Engle, R.W. (2005) Individual differences in working memory capacity and learning: evidence from the serial reaction time task. Mem. Cogn., 33, 213-220. 